\title{
Silibinin inhibits migration and invasion of the rhabdoid tumor G401 cell line via inactivation of the PI3K/Akt signaling pathway
}

\author{
YUMEI LI ${ }^{1}$, CHUNMEI ZHANG $^{2}$, DANFENG CAI $^{1}$, CONGDE CHEN $^{1}$ and DONGMEI MU ${ }^{3}$ \\ Departments of ${ }^{1}$ Pediatric Surgery, ${ }^{2}$ Nursing and ${ }^{3}$ Pediatric Neurosurgery, The Second Affiliated Hospital and \\ Yuying Children's Hospital of Wenzhou Medical University, Wenzhou, Zhejiang 325027, P.R. China
}

Received October 5, 2016; Accepted September 22, 2017

DOI: $10.3892 / 01.2017 .7246$

\begin{abstract}
Rhabdoid tumors, which tend to occur prior to the age of 2 years, are one of the most aggressive malignancies and have a poor prognosis due to the frequency of metastasis. Silibinin, a natural extract, has been approved as a potential tumor suppressor in various studies, however, whether or not it also exerts its antitumor capacity in rhabdoid tumors, particularly with regards to tumor migration and invasion, is unclear. The rhabdoid tumor G401 cell line was used in the present in vitro study. An MTT assay was used to assess the cytotoxicity of silibinin on G401 cells, cell migration was studied using a wound healing assay and a Transwell migration assay, and cell invasion was determined using a Transwell invasion assay. The underlying mechanism in silibinin inhibited cell migration and invasion was investigated by western blot analysis and further confirmed using a specific inhibitor. Experimental results demonstrated that high doses of silibinin suppressed cell viability, and that low doses of silibinin inhibited cell migration and invasion without affecting cell proliferation. The phosphatidylinositol 3-kinase/protein kinase B (PI3K/Akt) signaling pathway was involved in the silibinin-induced inhibition of metastasis. Silibinin inactivated the PI3K/Akt pathway, and inhibited cell migration and invasion, an effect that was further enhanced when LY294002, a classic PI3K inhibitor, was used concurrently. In general, silibinin inhibits migration and invasion of the rhabdoid tumor G401 cell line via inactivation of the PI3K/Akt signaling pathway and may be a potential chemotherapeutic drug to combat rhabdoid tumors in the future.
\end{abstract}

Correspondence to: Dr Dongmei Mu, Department of Pediatric Neurosurgery, The Second Affiliated Hospital and Yuying Children's Hospital of Wenzhou Medical University, 109, Xueyuan West Road, Wenzhou, Zhejiang 325027, P.R. China

E-mail: dongmeimu1973@gmail.com

Key words: rhabdoid tumor, silibinin, migration, invasion, phosphatidylinositol 3-kinase/Akt

\section{Introduction}

Rhabdoid tumors are the most common sarcoma derived from the soft tissues in patients under the age of 20 years. Approximately $10 \%$ of pediatric solid tumors are rhabdoid tumors, accounting for $\sim 50 \%$ of all rhabdoid tumor malignancies $(1,2)$. Proliferation and metastasis are two typical characteristics of rhabdoid tumors and $90 \%$ of affected patients are expected to succumb within 5 years. Surgery combined with systemic radiotherapy is recommended to promote survival, particularly in pediatric patients. However, amputation is unavoidable in certain circumstances and presents a substantial physical and psychological challenge to patients and their families (3-5). Proton radiotherapy provides hope to patients with rhabdoid tumors, as it efficiently inhibits tumor growth and metastasis without damaging normal tissues or impairing growth or development. However, it is difficult to widely utilize proton radiotherapy, particularly in developing countries, due to its limitations and high cost (6-8). Chemotherapy drugs, including Adriamycin and cyclophosphamide, have been trialed in certain cases, but their outcomes remain uncertain (8). Therefore, identification of effective and affordable drugs to cure rhabdoid tumors is required.

Silibinin is a natural extract obtained from milk thistle (Silybum marianum) that has been widely used in Asian counties as an anti-oxidant drug (9). One of its known functions is liver protection, as it stabilizes the cell membrane and reduces reactive oxygen species in liver cells $(10,11)$. In addition to its role in normal cell protection, silibinin has attracted more attention due to its potential antitumor capacities. Silibinin triggers apoptosis, induces cell cycle arrest or autophagic cell death to inhibit cell proliferation, and suppresses cell migration and invasion in a variety of cancer models, including breast, prostate, bladder and renal cancer types (12-15). Further investigation is required to determine whether or not silibinin inhibits rhabdoid tumors, particularly with regards to tumor cell migration and invasion.

The phosphatidylinositol 3-kinase/protein kinase B (PI3K/Akt) signaling pathway serves a pivotal function in cell homeostasis and has been revealed to be involved in cell proliferation and metastasis regulation $(16,17)$. Multiple studies have revealed that the PI3K/Akt signaling pathway is extensively activated in a variety of tumor types, including breast cancer, prostate cancer and rhabdoid tumors; inactivation of PI3K/Akt 
using specific inhibitors or other anticancer drugs leads to cell proliferation inhibition, migration and invasion suppression (18-21). Silibinin has been reported to be a PI3K/Akt suppressor and thus inhibit tumorigenesis and cancer progression $(22,23)$. Whether or not PI3K/Akt is also affected by silibinin in rhabdoid tumors is unclear and requires further clarification.

To the best of our knowledge, the present study was the first to evaluate the antitumor capacity of silibinin in rhabdoid tumors, with respect to cell viability, migration and invasion, with a specific focus on the inactivation of the PI3K/Akt signaling pathway.

\section{Materials and methods}

Reagents. Silibinin (cat. no. S0417), MTT (cat. no. M2128), protease inhibitor (cat. no. P8340) and phosphatase inhibitor (cat.no.MSSAFE) were purchased from Sigma-Aldrich (Merck KGaA, Darmstadt, Germany). The PI3K inhibitor LY294002 (cat. no. S1105) and proliferation inhibitor mitomycin C (cat. no. S8146) were purchased from Selleck Chemicals (Houston, TX, USA). Matrix gel (cat. no. 356234) was purchased from BD Biosciences (San Jose, CA, USA). The bicinchoninic acid (BCA) protein qualification kit (cat. no. 23225) was purchased from Pierce; Thermo Fisher Scientific, Inc. (Waltham, MA, USA). Anti-phosphorylated-Akt (Ser473; cat. no. 4060), anti-total (t)-Akt (cat. no. 4691), anti-GAPDH (cat. no. 5174) primary antibodies, and horseradish peroxidase (HRP)-conjugated goat anti-rabbit IgG (H+L) (cat. no. 7074) and HRP-conjugated horse anti-mouse $\operatorname{IgG}(\mathrm{H}+\mathrm{L})$ (cat. no. 7076) secondary antibodies were purchased from Cell Signaling Technology, Inc. (Danvers, MA, USA). Dilutions for primary antibodies and secondary antibodies were 1:1,000 and 1:10,000 respectively.

Cell culture. The human rhabdoid tumor G401 cell line was purchased from the American Type Culture Collection (cat. no. CRL-1441; ATCC, Manassas, VA, USA) and cultured in complete medium which contained ATCC-formulated McCoy's 5A modified medium (cat. no. 30-2007; ATCC) supplemented with $10 \%$ fetal bovine serum (FBS; cat. no. 10438026; Thermo Fisher Scientific, Inc.), $100 \mathrm{U} / \mathrm{ml}$ penicillin and $0.1 \mathrm{mg} / \mathrm{ml}$ streptomycin. Cells were incubated in a cell culture incubator (cat. no. 3308; Thermo Fisher Scientific, Inc.) with a humidified atmosphere containing $5 \% \mathrm{CO}_{2}$ at $37^{\circ} \mathrm{C}$. An inverted microscope at x40 magnification (Olympus, Tokyo, Japan) was used to observe cells and to capture images. When cells achieved 90-100\% confluency, cells were digested by $0.25 \%$ trypsin in $0.53 \mathrm{mM}$ EDTA solution and centrifuged at $100 \mathrm{x} \mathrm{g}$ for $5 \mathrm{~min}$ at room temperature. Cultured medium was replaced every other day or according to the experimental design.

Cell viability assay. An MTT assay was used to determine cell viability. A total of $200 \mu \mathrm{l}$ medium containing 3,000 cells was placed into each well of a 96 -well plate overnight in the cell culture incubator at $37^{\circ} \mathrm{C}$. The next day, culture medium was replaced with complete medium containing 10, 20 or $40 \mu \mathrm{M}$ silibinin or DMSO and cells were incubated in the cell culture incubator at $37^{\circ} \mathrm{C}$ for $24 \mathrm{~h}$. MTT was added to the medium to obtain a final concentration of $0.5 \mathrm{mg} / \mathrm{ml} 4 \mathrm{~h}$ prior to harvest. The medium was aspirated in each well and $150 \mathrm{ml}$ dimethylsulfoxide (DMSO) per well was added to dissolve the precipitates. The color change was then measured, and the optical density (OD) value was read by a plate reader at a wavelength of $490 \mathrm{~nm}$.

Wound healing assay. A 6-well plate was used to perform a wound-healing assay and each well was seeded with 500,000 cells in $2 \mathrm{ml}$ ATCC-formulated McCoy's 5A modified medium supplemented with $10 \%$ FBS, $100 \mathrm{U} / \mathrm{ml}$ penicillin and $0.1 \mathrm{mg} / \mathrm{ml}$ streptomycin. When the cell density reached $100 \%$ confluence, wounds were scratched using $200-\mu 1$ pipet tips in each well and were rinsed with $37^{\circ} \mathrm{C}$ preheated phosphate-buffered saline (PBS) 3 times. Cells were then cultured in $2 \mathrm{ml}$ ATCC-formulated McCoy's 5A modified medium supplemented with $100 \mathrm{U} / \mathrm{ml}$ penicillin and $0.1 \mathrm{mg} / \mathrm{ml}$ streptomycin containing $20 \mu \mathrm{M}$ silibinin, $10 \mu \mathrm{M}$ LY294002, both or vehicle (DMSO), and $1 \mu \mathrm{g} / \mathrm{ml}$ mitomycin $\mathrm{C}$ was added to each well. An inverted microscope at x40 magnification (Olympus, Tokyo, Japan) was used to image these wounds at designated time points, 12 and $24 \mathrm{~h}$.

Transwell migration and invasion assays. Cells were cultured in a 6-well plate and treated with $20 \mu \mathrm{M}$ silibinin, $10 \mu \mathrm{M}$ LY294002, both or vehicle (DMSO), once they had reached $60 \%$ confluence. After $24 \mathrm{~h}$ of treatment, the cells were digested and centrifuged at $100 \mathrm{x}$ g for $5 \mathrm{~min}$ at room temperature. A total of 30,000 cells were seeded onto a Millicell (cat. no. PSET010R1; Merck KGaA, Darmstadt, Germany) without matrix gel (migration assay) or 100,000 cells were seeded with matrix gel (invasion assay) in $200 \mu \mathrm{l}$ ATCC-formulated McCoy's 5A modified medium supplemented with $100 \mathrm{U} / \mathrm{ml}$ penicillin and $0.1 \mathrm{mg} / \mathrm{ml}$ streptomycin. The Millicells were then placed into the wells of a 12-well plate. The space inside Millicells was considered to be upper chamber, while the space between the bottom of Millicells and the bottom of the 12-well plate was considered to be lower chamber. The lower chamber of each well was filled with $800 \mu$ ATCC-formulated McCoy's $5 \mathrm{~A}$ modified medium supplemented with $10 \% \mathrm{FBS}, 100 \mathrm{U} / \mathrm{ml}$ penicillin and $0.1 \mathrm{mg} / \mathrm{ml}$ streptomycin, prior to being placed into the cell culture incubator for $24 \mathrm{~h}$ to allow cells to migrate or invade. When the Millicells were harvested, medium was aspirated, and the membranes from the inserts were washed with $4^{\circ} \mathrm{C}$ PBS 3 times, fixed with $4 \%$ paraformaldehyde and stained with $0.1 \%$ crystal violet at room temperature for $15 \mathrm{~min}$ respectively. The membranes were then cut and placed onto slides. Migrated and invaded cells were then observed and images were captured using a bright-field light microscope (at x100 magnification; Olympus, Tokyo, Japan).

Western blot analysis. Treated cells were washed with pre-chilled $\left(4^{\circ} \mathrm{C}\right)$ PBS 3 times prior to being solubilized by radioimmunoprecipitation assay buffer (cat. no. 89900; Thermo Fisher Scientific, Inc.) containing protease inhibitor and phosphatase inhibitor. Lysate was then sonicated and centrifuged at $14,000 \mathrm{x}$ f for $10 \mathrm{~min}$ at $4^{\circ} \mathrm{C}$. The supernatant of each lysate was placed into a different tube, qualified by the BCA qualification system and boiled with $4 \mathrm{X}$ loading buffer (cat. no. NP0007; Thermo Fisher Scientific, Inc.). In total, $30 \mu \mathrm{g}$ 


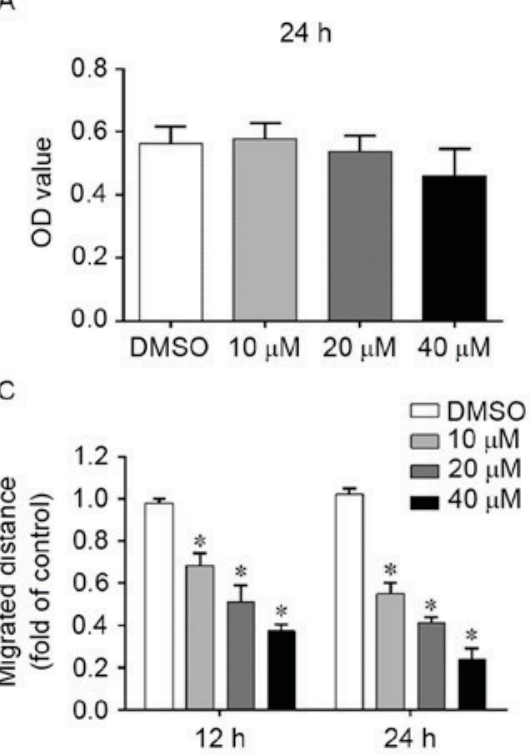

B

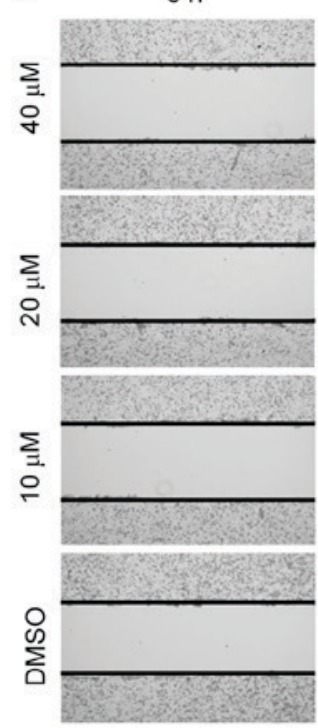

$12 \mathrm{~h}$

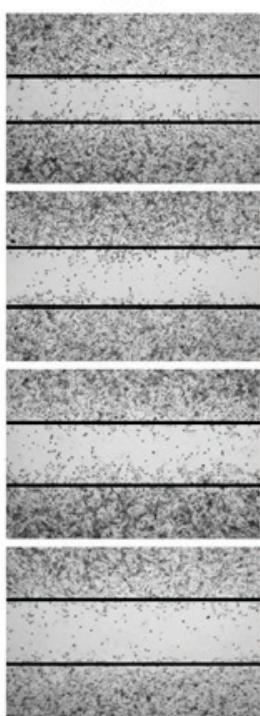

$24 \mathrm{~h}$

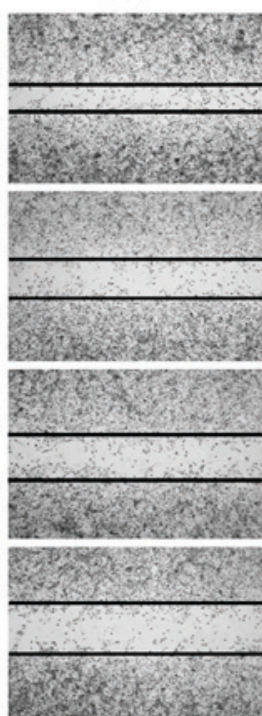

D
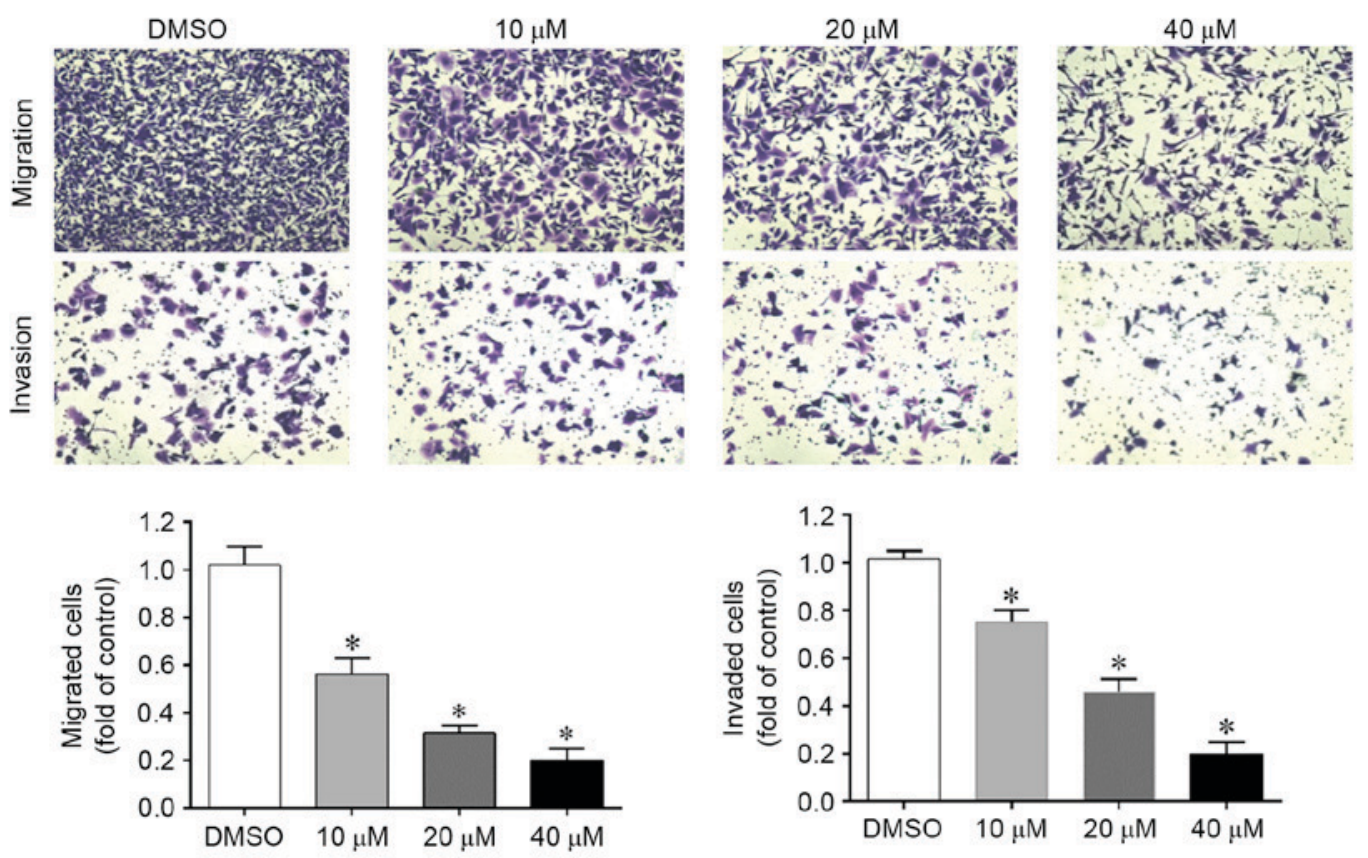

Figure 1. Silibinin inhibits migration and invasion of G401 in a concentration-dependent manner. (A) Cells were treated with silibinin (10,20 and $40 \mu \mathrm{M}$ ) or vehicle (DMSO) for $24 \mathrm{~h}$ and cell viability was confirmed using an MTT assay. (B and C) Cells were treated with silibinin (10,20 and $40 \mu \mathrm{M})$ or vehicle (DMSO) and cell migration was confirmed using a wound healing assay at 12 and $24 \mathrm{~h}$. (D) Cells were treated with silibinin (10, 20 and $40 \mu \mathrm{M})$ or vehicle (DMSO) for $24 \mathrm{~h}$ and then Transwell migration and invasion assays were performed. " $\mathrm{P}<0.05 \mathrm{vs}$. DMSO control. OD, optical density.

protein of each sample was loaded onto each column in $12 \%$ SDS-PAGE gel and electrophoresis was performed. Proteins were subsequently transferred onto a polyvinylidene fluoride membrane. Membranes were blocked with 5\% milk at room temperature for $1 \mathrm{~h}$ and incubated with primary antibodies for $2 \mathrm{~h}$ at room temperature or overnight at $4^{\circ} \mathrm{C}$. Membranes were washed with Tris-buffered saline plus $0.1 \%$ Tween (TBST) and subsequently incubated with secondary antibodies for $1 \mathrm{~h}$ at room temperature. Bands in the membranes were visualized using ECL reagents (cat. no. 1705060; Bio-Rad Laboratories, Inc.) and were detected by ChemiDoc ${ }^{\mathrm{TM}} \mathrm{XRS}+$ system (Bio-Rad Laboratories, Inc.). The density of the bands was quantified by ImageJ software (version $1.50 \mathrm{~g} 13$; National Institutes of Health, Bethesda, MD, USA).
Statistical analysis. All experiments were performed $\geq 3$ times. GraphPad Prism 5 (GraphPad software, La Jolla, CA, USA) was utilized to generate graphs. Data are presented as the mean \pm standard deviation and the differences between experimental groups were analyzed using one-way analysis of variance by SPSS 17.0 (IBM, Chicago, IL, USA). P<0.05 was considered to indicate a statistically significant difference.

\section{Results}

Silibinin inhibits migration and invasion of G401 cells in a dose-dependent manner. G401 is a well-established cell line derived from the kidney of a 3-month-old male patient. It was first classified as a Wilm's tumor cell line, but this was 
A

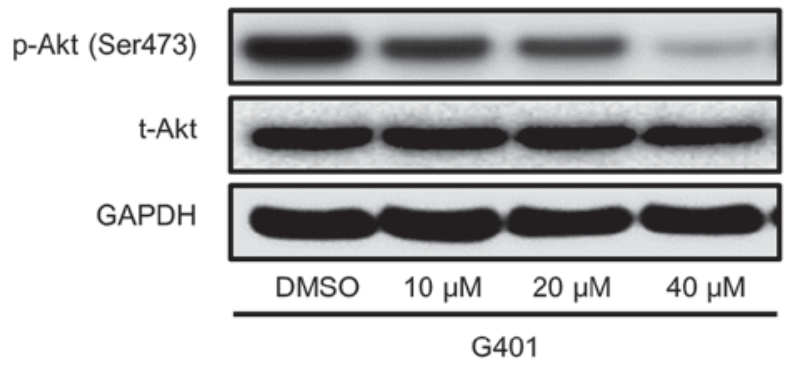

B

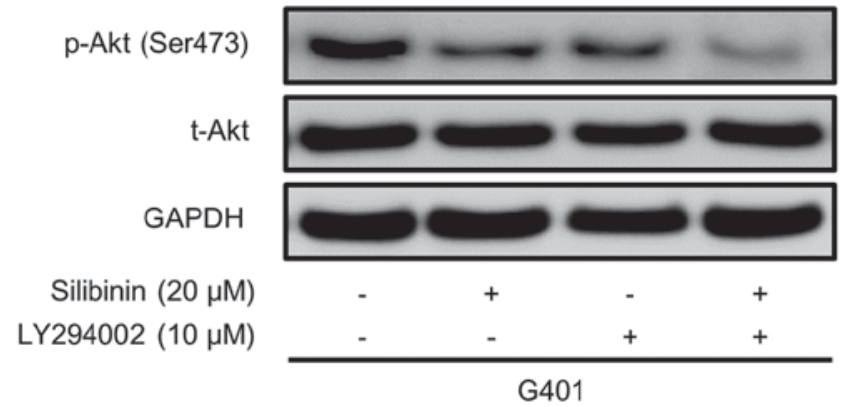

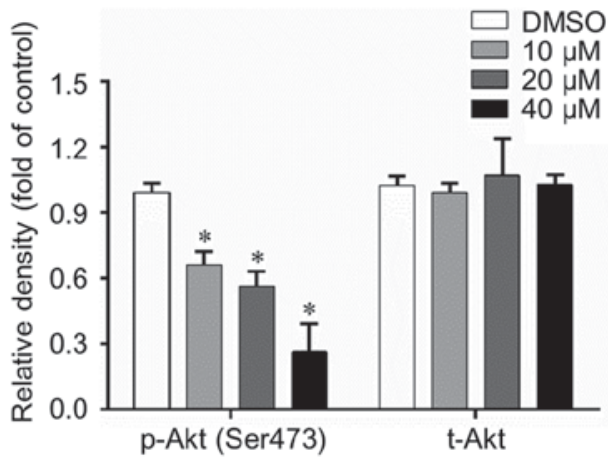

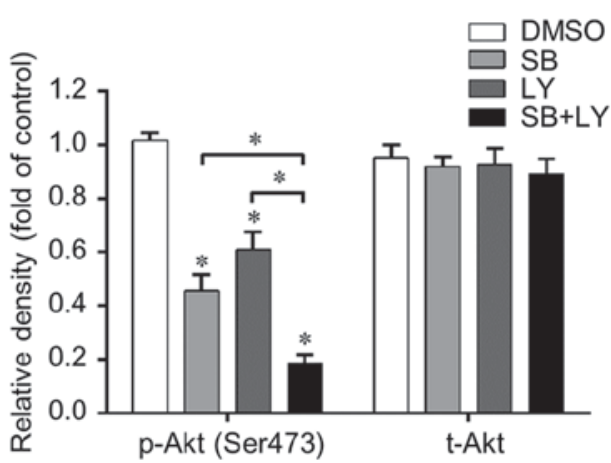

Figure 2. PI3K/Akt signaling pathway is inactivated by silibinin in G401 cells. (A) Cells were treated with silibinin (10, 20 and $40 \mu \mathrm{M}$ ) or vehicle (DMSO) for $24 \mathrm{~h}$ and harvested by radioimmunoprecipitation assay lysis buffer. p-Akt (Ser473) and t-Akt were confirmed by western blot analysis. (B) Cells were treated

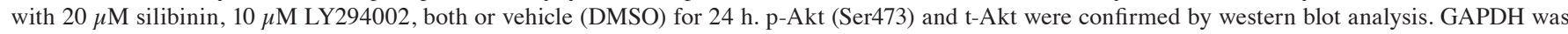
used as the loading control. "P<0.05 vs. control unless otherwise stated. PI3K/Akt, phosphatidylinositol 3-kinase/protein kinase B; p-, phosphorylated; t-, total.

subsequently corrected and it is now known to be a rhabdoid tumor cell line (24). This cell line has been utilized by several groups and has been proven to be an ideal in vitro model for studying rhabdoid tumors (25-27). However, whether or not the oncological behavior of G401 cells can be affected by silibinin remains unclear.

Low doses of silibinin have been demonstrated to inhibit cell migration and invasion in various tumor models in vivo and in vitro. In the present study, an MTT assay was initially used to select the doses that did not significantly affect cell growth over a treatment period of $24 \mathrm{~h}$. As demonstrated in Fig. 1A, when cells were treated with 10,20 or $40 \mu \mathrm{M}$ silibinin for $24 \mathrm{~h}$, cell viability was unaffected compared with the DMSO control and thus, doses were chosen to be used in subsequent studies. High doses of silibinin and longer exposure times were revealed to significantly decrease cell viability (data not shown).

A wound healing assay is a convenient, economic and reliable assay for studying cell migration. Therefore, in order to evaluate whether silibinin affects the migration of G401 cells, a wound healing assay was performed. As demonstrated in Fig. 1B and C, when the cells were treated with silibinin, they migrated more slowly as the wounds had less closure. To further confirm this finding, a Transwell migration assay was performed and similar results were obtained (Fig. 1D).

To study the impact of silibinin on cell invasion, a Transwell invasion assay was performed. In this assay, cells will invade the other side of the membrane only when the pre-coated Matrigel is digested by the cells themselves. As demonstrated in Fig. 1D, silibinin significantly inhibited cell invasion in a concentration-dependent manner.

These results indicate that silibinin inhibits G401 migration and invasion independent of its growth inhibition ability.

PI3K/Akt signaling pathway is inactivated by silibinin in G401. The PI3k/Akt signaling pathway serves a pivotal function in cell homeostasis and is a crucial regulator of cell growth, migration and invasion. Whether or not this pathway is involved in the silibinin-induced inhibition of migration and invasion remains unclear. Therefore, western blot analysis was performed in order to confirm this hypothesis. As presented in Fig. 2A, p-Akt (Ser473) was suppressed by silibinin, while t-Akt was not significantly affected. To further confirm the function of the PI3k/Akt pathway, LY294002, a classic PI3K inhibitor, was used. LY294002 and silibinin independently inhibited the activation of p-Akt without affecting the total level of Akt. When these two reagents were used in combination, the PI3K/Akt inhibition effect was further enhanced (Fig. 2B). These results suggest that the PI3K/Akt signaling pathway is suppressed when cells are treated with silibinin.

PI3K/Akt signaling pathway is involved in the inhibition of migration and invasion induced by silibinin. Since silibinin was confirmed to be a PI3K/Akt suppressor in the G401 cell line and as silibinin inhibits the migration and invasion of G401 cells, the role of PI3K/Akt inactivation in the silibinin-induced inhibition of migration and invasion requires further investigation. In the wound healing assay, silibinin and LY294002 independently suppressed wound closure, 
A

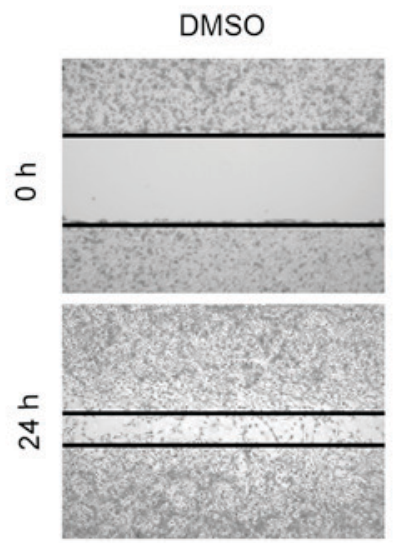

$\mathrm{SB}$
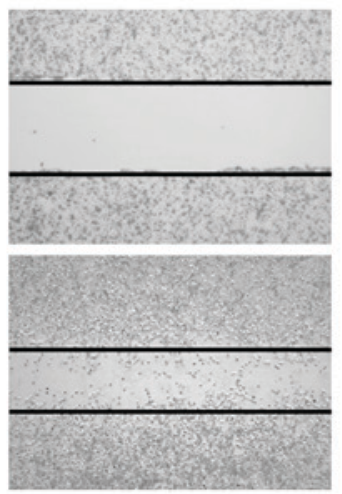

LY
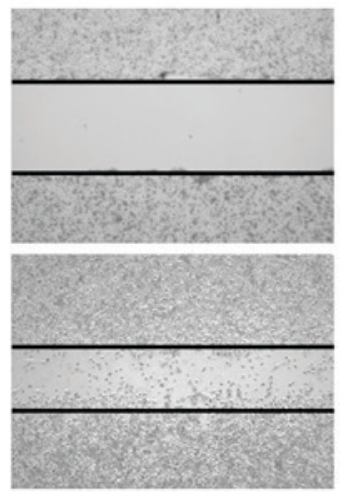

$S B+L Y$

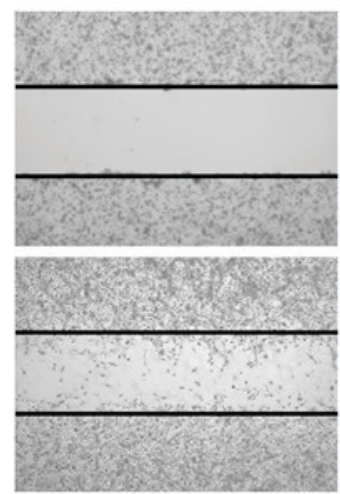

B

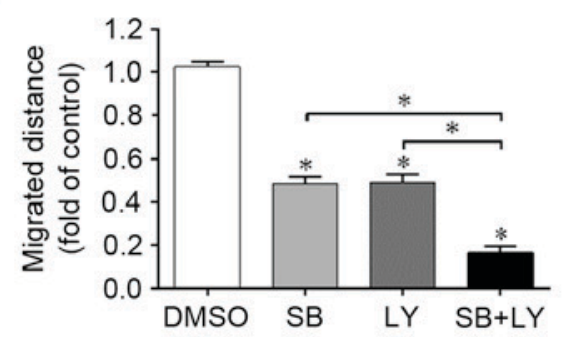

C
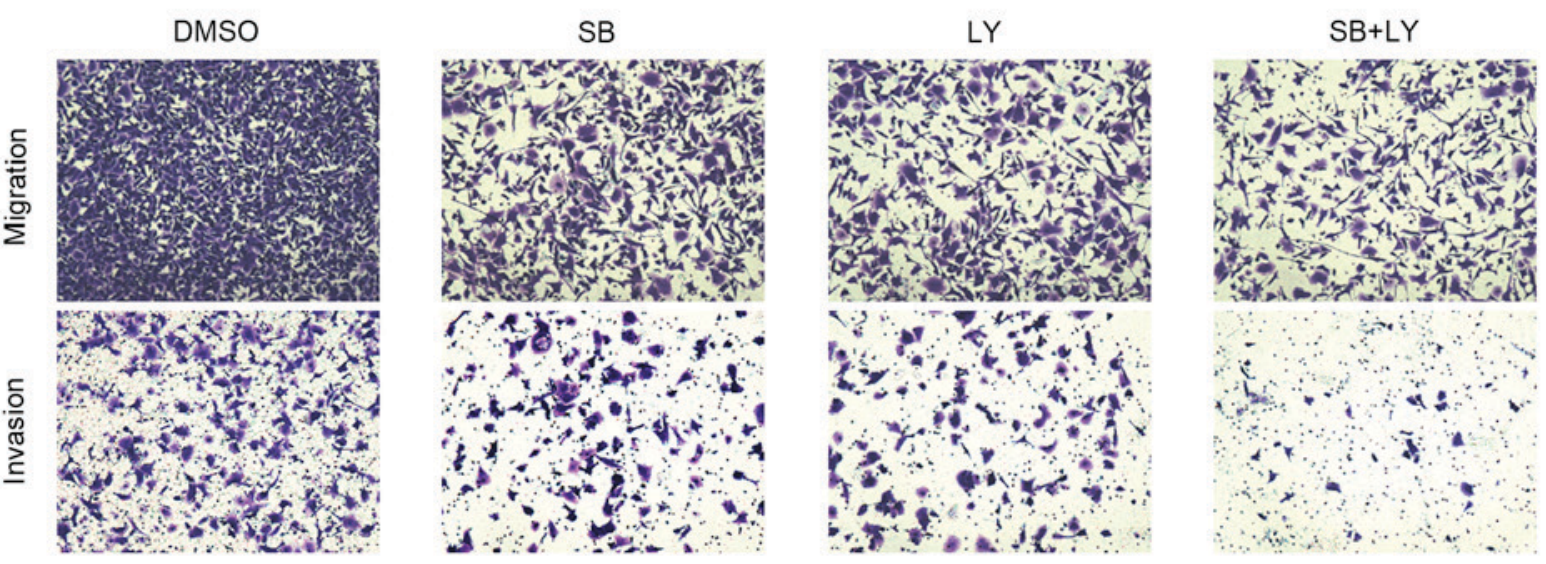

D
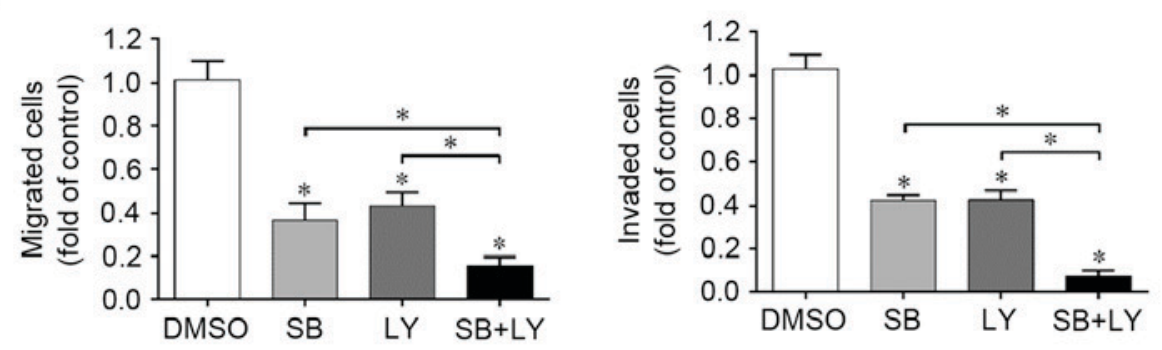

Figure 3. PI3K/Akt signaling pathway is involved in the silibinin-induced inhibition of migration and invasion. Cells were treated with $20 \mu \mathrm{M}$ silibinin, $10 \mu \mathrm{M}$ LY294002, both or vehicle (DMSO). Cell migration was assessed by (A and B) wound healing assay and (C and D) Transwell migration and invasion assays were used to confirm cell invasion and migration. "P<0.05 vs. control unless otherwise stated. PI3K/Akt, phosphatidylinositol 3-kinase/protein kinase B; SB, silibinin; LY, LY294002.

and when these two reagents were used in combination, the inhibitory effect was enhanced (Fig. 3A and B). These results were further confirmed using a Transwell migration assay (Fig. 3C and D).
To illustrate the role served by PI3K/Akt in silibinin-induced invasion inhibition, the Transwell invasion assay was performed again. As presented in Fig. 3C and D, silininin and LY294002 suppressed cell invasion, as there were fewer 
invaded cells in these groups than in the control group. Furthermore, when these two compounds were used in combination, cell invasion was further inhibited as this group had the least invaded cells. These data suggest that the inactivation of PI3K/Akt by silibinin positively contributes to the inhibition of cell migration and invasion.

\section{Discussion}

Rhabdoid tumors, which usually occur in individuals under the age of 2 years, are one of the most aggressive malignancies and present with a poor prognosis. Surgery combined with systemic radiotherapy is currently the recommended treatment, but the efficiency of this method is limited due to the high metastatic capacity of rhabdoid tumors (3-5). Therefore, metastasis suppression, together with proliferation inhibition, is the key to curing rhabdoid tumors. Silibinin, a natural extract, has been approved as a potential tumor suppressor in various in vivo and in vitro studies (13). However, whether or not silibinin also exerts its antitumor capacity in rhabdoid tumors is unclear. To the best of our knowledge, the present in vitro study was the first to identify that silibinin inhibits proliferation, migration and invasion in the rhabdoid tumor cell line, G401, via inactivation of the PI3K/Akt signaling pathway.

Numerous studies have indicated that silibinin is a powerful antimetastatic drug; it inhibits $\beta$-catenin/ZEB1 signaling, epithelial-mesenchymal transition and stemness in bladder cancer in order to suppress metastasis (28). In renal cancer, mitogen-activated protein kinases are inactivated by silibinin, thereby suppressing cell migration and invasion (29,30). In the present study, in G401, a rhabdoid tumor cell line, silibinin was revealed to significantly inhibit cell migration in a wound healing assay and a Transwell migration assay, and cell invasion in a Transwell invasion assay in a concentration-dependent manner.

The PI3K/Akt pathway is an important intracellular signaling pathway that is directly associated with cellular quiescence, proliferation and metastasis. PI3K is enhanced by various factors, including, epidermal growth factor and insulin, and is antagonized by several oncoproteins, including phosphatase and tensin homolog $(17,21)$. PI3K activation facilities Akt phosphorylation and activation, localizes it at the plasma membrane, and thereby genetically and epigenetically affects its downstream target genes (31-34). This pathway is known to be overactive in a number of cancer types, resulting in reduced apoptosis and facilitated proliferation and metastasis, and is therefore considered to be a potential therapeutic target. Inactivation of the PI3K/Akt pathway in breast cancer induces apoptosis, cell cycle arrest and autophagy to suppress cell proliferation $(19,35)$. Meanwhile, epithelial-mesenchymal transition and matrix metalloproteinase synthesis are reduced by $\mathrm{PI} 3 \mathrm{~K}$ antagonists, and migration and invasion are inhibited as a result $(36,37)$. Additionally, inhibition of the PI3K/Akt pathway sensitizes cancer cells to chemotherapeutic drugs, including, doxorubicin (38).

The precise function served by the PI3K/Akt pathway in rhabdoid tumors is unclear since studies regarding this are limited, but it is likely to be overactivated, as it has been reported to be a dependent factor for cell survival (39). Silibinin has been proven to be an efficient PI3K/Akt pathway inhibitor; however, whether or not it affects this signaling pathway in rhabdoid tumors and contributes to their anti-metastatic ability requires further elucidation. The present study revealed that, following administration of silibilin, the PI3K/Akt signaling pathway was inactivated and this positively contributed towards the inhibition of metastasis. Silibinin and LY294002 independently inhibited the PI3K/Akt pathway, migration and invasion. When these two compounds were used in combination, PI3K/Akt signaling suppression and metastasis inhibition were further enhanced. Nevertheless, the present study did not investigate the manner through which silibinin affects $\mathrm{PI} 3 \mathrm{~K} / \mathrm{Akt}$ signaling and thus, further studies are required in order to determine this.

In addition to inhibiting metastasis, the present study also observed that silibinin suppressed G401 cell viability at high doses and longer exposure times. Silibinin has been demonstrated to be a strong proliferation suppressor via its ability to initiate and activate multiple programs, including, apoptosis and cell cycle arrest, in various cancer models, but whether or not these are involved in silibinin-induced growth inhibition in rhabdoid tumors requires further investigation.

Taken together, the results of the present in vitro study indicate that silibinin inhibits migration and invasion of the rhabdoid tumor cell line, G401, and that it inactivates the PI3K/Akt signaling pathway. These findings contribute toward the understanding of the antitumor capacity of silibinin and suggest that silibinin may be a potential chemotherapeutic drug for combating rhabdoid tumors in the future.

\section{Acknowledgements}

The present study was supported by the Foundation of Health and Family Planning Commission of Zhejiang Province (grant no. 20146242) and the Foundation of Wenzhou Science and Technology Bureau (grant no. Y20140248).

\section{References}

1. Siegel RL, Miller KD and Jemal A: Cancer statistics, 2016: CA Cancer J Clin 66: 7-30, 2016.

2. Miller KD, Siegel RL, Lin CC, Mariotto AB, Kramer JL, Rowland JH, Stein KD, Alteri R and Jemal A: Cancer treatment and survivorship statistics, 2016. CA Cancer J Clin 66: 271-289, 2016.

3. Geller JI: Current standards of care and future directions for 'high-risk' pediatric renal tumors: Anaplastic Wilms tumor and Rhabdoid tumor. Urol Oncol 34: 50-56, 2016.

4. Kuroda N, Karashima T, Inoue K, Kasajima A, Ohe C, Kawakami F, Mikami S, Matsuura K, Moriyama M, Nagashima Y, et al: Review of renal cell carcinoma with rhabdoid features with focus on clinical and pathobiological aspects. Pol J Pathol 66: 3-8, 2015.

5. National Cancer Institute: Childhood Central Nervous System Atypical Teratoid/Rhabdoid Tumor Treatment (PDQ $\left.{ }^{\circledR}\right)$. Health professional version, 2002.

6. McGovern SL, Okcu MF, Munsell MF, Kumbalasseriyil N, Grosshans DR, McAleer MF, Chintagumpala M, Khatua S and Mahajan A: Outcomes and acute toxicities of proton therapy for pediatric atypical teratoid/rhabdoid tumor of the central nervous system. Int J Radiat Oncol Biol Phys 90: 1143-1152, 2014.

7. Kralik SF, Ho CY, Finke W, Buchsbaum JC, Haskins CP and Shih CS: Radiation necrosis in pediatric patients with brain tumors treated with proton radiotherapy. AJNR Am J Neuroradiol 36: 1572-1578, 2015.

8. Elsayad K, Kriz J, Samhouri L, Haverkamp U, Straeter R, Stummer W and Eich HT: Long-term survival following additive radiotherapy in patients with atypical teratoid rhabdoid tumors. Strahlenther Onkol 192: 569-581,2016. 
9. Wellington K and Jarvis B: Silymarin: A review of its clinical properties in the management of hepatic disorders. BioDrugs 15: 465-489, 2001

10. Sozen H, Celik OI, Cetin ES, Yilmaz N, Aksozek A, Topal Y, Cigerci IH and Beydilli H: Evaluation of the protective effect of silibinin in rats with liver damage caused by itraconazole. Cell Biochem Biophys 71: 1215-1223, 2015.

11. Flora K, Hahn M, Rosen H and Benner K: Milk thistle (Silybum marianum) for the therapy of liver disease. Am J Gastroenterol 93: $139-143,1998$

12. Dastpeyman M, Motamed N, Azadmanesh K, Mostafavi E, Kia V, Jahanian-Najafabadi A and Shokrgozar MA: Inhibition of silibinin on migration and adhesion capacity of human highly metastatic breast cancer cell line, MDA-MB-231, by evaluation of $\beta 1$-integrin and downstream molecules, Cdc42, Raf-1 and D4GDI. Med Oncol 29: 2512-2518, 2012.

13. Vue B, Zhang S, Zhang X, Parisis K, Zhang Q, Zheng S, Wang $\mathrm{G}$ and Chen QH: Silibinin derivatives as anti-prostate cancer agents: Synthesis and cell-based evaluations. Eur J Med Chem 109: 36-46, 2016

14. Zeng J, Sun Y, Wu K, Li L, Zhang G, Yang Z, Wang Z, Zhang D, Xue Y, Chen Y, et al: Chemopreventive and chemotherapeutic effects of intravesical silibinin against bladder cancer by acting on mitochondria. Mol Cancer Ther 10: 104-116, 2011.

15. Li F, Ma Z, Guan Z, Chen Y, Wu K, Guo P, Wang X, He D and Zeng J: Autophagy induction by silibinin positively contributes to its anti-metastatic capacity via AMPK/mTOR pathway in renal cell carcinoma. Int J Mol Sci 16: 8415-8429, 2015.

16. Massacesi C,Di Tomaso E, Urban P,Germa C,QuadtC, Trandafir L, Aimone P, Fretault N, Dharan B, Tavorath R and Hirawat S: PI3K inhibitors as new cancer therapeutics: Implications for clinical trial design. Onco Targets Ther 9: 203-210, 2016.

17. Faes S and Dormond O: PI3K and AKT: Unfaithful partners in cancer. Int J Mol Sci 16: 21138-21152, 2015.

18. Mundi PS, Sachdev J, McCourt C and Kalinsky K: AKT in cancer: New molecular insights and advances in drug development. Br J Clin Pharmacol 82: 943-956, 2016.

19. Yang SX, Polley E and Lipkowitz S: New insights on PI3K/AKT pathway alterations and clinical outcomes in breast cancer. Cancer Treat Rev 45: 87-96, 2016.

20. Chen H, Zhou L, Wu X, Li R, Wen J, Sha J and Wen X: The $\mathrm{PI} 3 \mathrm{~K} / \mathrm{AKT}$ pathway in the pathogenesis of prostate cancer. Front Biosci (Landmark Ed) 21: 1084-1091, 2016.

21. Foster K, Wang Y, Zhou D and Wright C: Dependence on $\mathrm{PI} 3 \mathrm{~K} / \mathrm{Akt}$ signaling for malignant rhabdoid tumor cell survival. Cancer Chemother Pharmacol 63: 783-791, 2009.

22. Zhang Y, Hai J, Cao M, Zhang Y, Pei S, Wang J and Zhang Q: Silibinin ameliorates steatosis and insulin resistance during non-alcoholic fatty liver disease development partly through targeting IRS-1/PI3K/Akt pathway. Int Immunopharmacol 17 714-720, 2013

23. Feng N, Luo J and Guo X: Silybin suppresses cell proliferation and induces apoptosis of multiple myeloma cells via the PI3K/Akt/mTOR signaling pathway. Mol Med Rep 13: 3243-3248, 2016

24. Garvin AJ, Re GG, Tarnowski BI, Hazen-Martin DJ and Sens DA: The G401 cell line, utilized for studies of chromosomal changes in Wilms' tumor, is derived from a rhabdoid tumor of the kidney. Am J Pathol 142: 375-380, 1993.

25. Krust B, El Khoury D, Soundaramourty C, Nondier I and Hovanessian AG: Suppression of tumorigenicity of rhabdoid tumor derived G401 cells by the multivalent HB-19 pseudopeptide that targets surface nucleolin. Biochimie 93: 426-433, 2011.
26. Unland R, Borchardt C, Clemens D, Kool M, Dirksen U and Frühwald MC: Analysis of the antiproliferative effects of 3-deazaneoplanocin A in combination with standard anticancer agents in rhabdoid tumor cell lines. Anticancer Drugs 26: 301-311, 2015.

27. Megison ML, Gillory LA, Stewart JE, Nabers HC, Mrozcek-Musulman E and Beierle EA: FAK inhibition abrogates the malignant phenotype in aggressive pediatric renal tumors. Mol Cancer Res 12: 514-526, 2014

28. Wu K, Ning Z, Zeng J, Fan J, Zhou J, Zhang T, Zhang L, Chen Y, Gao Y, Wang B, et al: Silibinin inhibits $\beta$-catenin/ZEB1 signaling and suppresses bladder cancer metastasis via dual-blocking epithelial-mesenchymal transition and stemness. Cell Signal 25: 2625-2633, 2013.

29. Chang HR, Chen PN, Yang SF, Sun YS, Wu SW, Hung TW, Lian JD, Chu SC and Hsieh YS: Silibinin inhibits the invasion and migration of renal carcinoma 786-O cells in vitro, inhibits the growth of xenografts in vivo and enhances chemosensitivity to 5-fluorouracil and paclitaxel. Mol Carcinog 50: 811-823, 2011.

30. Liang L, Li L, Zeng J, Gao Y, Chen YL, Wang ZQ, Wang XY, Chang LS and He D: Inhibitory effect of silibinin on EGFR signal-induced renal cell carcinoma progression via suppression of the EGFR/MMP-9 signaling pathway. Oncol Rep 28: 999-1005, 2012.

31. King D, Yeomanson D and Bryant HE: PI3King the lock: Targeting the PI3K/Akt/mTOR pathway as a novel therapeutic strategy in neuroblastoma. J Pediatr Hematol Oncol 37: 245-251, 2015.

32. Wyatt LA, Filbin MT and Keirstead HS: PTEN inhibition enhances neurite outgrowth in human embryonic stem cell-derived neuronal progenitor cells. J Comp Neurol 522: 2741-2755, 2014.

33. Chen X, Wang YW, Xing AY, Xiang S, Shi DB, Liu L, Li YX and Gao P: Suppression of SPIN1-mediated PI3K-Akt pathway by miR-489 increases chemosensitivity in breast cancer. J Pathol 239: 459-472, 2016.

34. Pulido R: PTEN: A yin-yang master regulator protein in health and disease. Methods 77-78: 3-10, 2015.

35. Grunt TW and Mariani GL: Novel approaches for molecular targeted therapy of breast cancer: Interfering with PI3K/AKT/mTOR signaling. Curr Cancer Drug Targets 13: 188-204, 2013

36. Rafael D, Doktorovová S, Florindo HF, Gener P, Abasolo I, Schwartz S Jr and Videira MA: EMT blockage strategies: Targeting Akt dependent mechanisms for breast cancer metastatic behaviour modulation. Curr Gene Ther 15: 300-312, 2015.

37. Zhou R, Xu L, Ye M, Liao M, Du H and Chen H: Formononetin inhibits migration and invasion of MDA-MB-231 and 4T1 breast cancer cells by suppressing MMP-2 and MMP-9 through PI3K/AKT signaling pathways. Horm Metab Res 46: 753-760, 2014.

38. Chen WC, Lai YA, Lin YC, Ma JW, Huang LF, Yang NS, Ho CT, Kuo SC and Way TD: Curcumin suppresses doxorubicin-induced epithelial-mesenchymal transition via the inhibition of TGF- $\beta$ and PI3K/AKT signaling pathways in triple-negati breast cancer cells. J Agric Food Chem 61: 11817-11824, 2013.

39. Saletta F, Wadham C, Ziegler DS, Marshall GM, Haber M, McCowage G, Norris MD and Byrne JA: Molecular profiling of childhood cancer: Biomarkers and novel therapies. BBA Clin 1: 59-77, 2014 